Mappemonde

Revue trimestrielle sur l'image géographique et les

formes du territoire

$131 \mid 2021$

Varia

\title{
Présentation du concours GéoDataDays 2020
}

\section{Christine Plumejeaud}

URL : https://journals.openedition.org/mappemonde/6384

DOI : 10.4000/mappemonde.6384

ISSN : $1769-7298$

Éditeur

UMR ESPACE

\section{Référence électronique}

Christine Plumejeaud, «Présentation du concours GéoDataDays 2020 », Mappemonde [En ligne], $131 \mid$

2021, mis en ligne le 08 juillet 2021, consulté le 14 juillet 2021. URL : http://journals.openedition.org/ mappemonde/6384 ; DOI : https://doi.org/10.4000/mappemonde.6384

Ce document a été généré automatiquement le 14 juillet 2021.

\section{(c) (i) (2)(2)}

La revue Mappemonde est mise à disposition selon les termes de la Licence Creative Commons Attribution - Pas d'Utilisation Commerciale - Partage dans les Mêmes Conditions 4.0 International. 


\section{Présentation du concours GéoDataDays 2020}

Christine Plumejeaud 
1 Les GéoDataDays constituent un événement national indépendant dédié à la géographie numérique en France. Ces rencontres sont organisées par l'AFIGÉO et DécryptaGéo, en partenariat avec une plateforme régionale d'information géographique et des collectivités territoriales. L'édition 2020 qui s'est tenue à Montpellier les 15 et 16 septembre a permis d'organiser un concours en partenariat avec le GdR CNRS MAGIS pour récompenser de jeunes chercheurs (étudiants, doctorants ou post-doctorants) qui innovent en géomatique (https://www.geodatadays.fr/page/Ils-sont-candidats-auxChallenges-Geodata/56).

- Sivakavi Kumarasamy, élève de l'ENSIIE (École Nationale Supérieure d'Informatique pour l'Industrie et l'Entreprise) en apprentissage à l'IGN, a obtenu le premier prix avec OPIUM (https://journals.openedition.org/mappemonde/6343). Cette plateforme de simulation ludique de risques naturels, qui s'adresse aux enfants de primaire et du collègue, facilite l'appropriation de l'espace géographique grâce à l'usage conjoint de la carte 2D et la représentation 3D. L'intégration de données géographiques «à la carte » dans un moteur de simulation 3D est un atout majeur de ce « jeu sérieux » qui offre de belles perspectives aux politiques de sensibilisation aux risques par la simulation participative.

- Aline Menin est lauréate du concours. Elle a obtenu le deuxième prix pour le projet eSTIMe qui a suscité l'intérêt des professionnels présents à la manifestation (https:// journals.openedition.org/mappemonde/6349). Cet environnement de géovisualisation des mobilités quotidiennes propose, en effet, un design adaptatif et une esthétique remarquable et il est réutilisable pour d'autres données de flux.

- Thomas Maillard, géographe post-doctorant à l'UMR Passages, chercheur associé au LADYSS, est lauréat du concours avec un troisième prix qui récompense la pertinence de cet atlas interactif, réalisé en partenariat avec l'ONG Urgence Eau sur le sujet sensible de l'accès à l'eau potable en Côte d'Ivoire (https://journals.openedition.org/mappemonde/6268). Il a suscité l'engouement des visiteurs, qui lui décernent le prix du public. Cet outil fait en effet la démonstration de ce que peut apporter un atlas accessible à tous, avec un contenu pertinent, pour l'aide à la décision et la concertation autour d'un enjeu fort.

\section{AUTEUR}

\section{CHRISTINE PLUMEJEAUD}

Ingénieure de recherche, responsable Plateforme DISA (Données : Intégration, Structuration et Analyse). LIENSs (Littoral Environnement et Sociétés), UMR 7266 